\title{
Evaluation of filter paper collection of urine samples for detection and measurement of organic acidurias by capillary electrophoresis
}

\author{
Coral Barbas*, Antonia García, Laura de Miguel, Carolina Simó \\ Universidad San Pablo-CEU, Facultad de CC Experimentales y Técnicas Urbanización Montepríncipe, Ctra. Boadilla del Monte, \\ km 5.3, 28668 Boadilla del Monte, Madrid, Spain
}

Received 14 May 2001; received in revised form 7 June 2002; accepted 13 June 2002

\begin{abstract}
There is little doubt that mental retardation has been prevented in most babies diagnosed by newborn screening programs for inborn errors and the cost-benefit ratios of these programs have been reported as highly positive. In a previous work we optimised a CE method for quick profiling of organic acidurias, which characterize a large number of inborn errors, so that it permits the separation, detection and even identification in less than $15 \mathrm{~min}$ of 22 organic acids in urine samples related to a wide range of metabolic disorders. In the present work we have studied the adequacy of filter paper collection of urine samples to simplify this step, always difficult in babies, when it is not performed by training personnel. The studied parameters were: media and conditions for re-extraction to give the best sensitivity and a more simple procedure when the samples are measured by $\mathrm{CE}$, interferences coming from the diaper, recoveries obtained, possible correction of recoveries with creatinine and stability of the compounds. The whole method we report has the advantages of easy sample collection, easy shipping or delivery, and rapid analysis. Moreover, this method of collection and analysis allows the identification and quantitation of fumaric, methylmalonic, $N$-acetylaspartic, pyroglutamic and homogentisic acids, as well as glutaric acid for which screening is considered especially advisable.
\end{abstract}

(C) 2002 Elsevier Science B.V. All rights reserved.

Keywords: Organic acidurias; Filter paper collection

\section{Introduction}

Screening of infant urine for different pathologies has been conducted in several programs. The use of absorbent filter paper to collect urine for assays of homovanillic (HVA) and vanylmandelic (VMA) acids was reported by several authors [1-6]. Mass screening of organic acids is not so frequent, but

*Corresponding author. Fax: +34-91-351-0475.

E-mail address: cbarbas@ceu.es (C. Barbas). there are also several works reporting the use of filter paper collection of urine samples for detecting these pathologies: methylmalonic aciduria with analysis by GC-MS [7]; methylmalonic, and several aminoacids analysed by TLC [8]; methylmalonic and hydroxyphenyllactic determined by GC-MS; orotic acid measured by GC-MS [9]; multiple organic acidurias analysed by GC-MS [10] and "all" the acidic metabolites by GC-FID [11].

In their systematic review of newborn screening for inborn errors of metabolism, Seymour et al. [12] 
reported that most disorders of organic acid metabolism are diagnosed by GC-MS analysis. The method, which requires extraction of the organic acids and conversion into thermally and chemically stable derivatives, is quite unsuitable for large-scale newborn screening.

Tandem mass spectrometry (MS-MS) is the most important development in newborn screening since the addition of hypothyroidism in the mid-1970s [13]. Between 20 and 25 disorders are screened in a blood spot, but, nowadays, with few exceptions, health department screening programs do not have and are unlikely to be able to acquire and retain the equipment and expertise required to perform adequately MS-MS analyses and interpret the results.

Capillary electrophoresis (CE) is being admitted as a powerful tool for diagnostic applications suitable for detecting important changes in the metabolic profiles of body fluids [14-17]. Although when it is not coupled to an MS detector it has not the identification capabilities of the previously quoted techniques, it is easy to include in routine analytical laboratories and it can offer a rapid and simple alternative to other more sophisticated and expensive techniques, mainly for a first screening of the pathologies included in the study and for monitoring the therapy once the baby has been diagnosed.

It is unquestionable that MS-related techniques are better tools for wide-ranging screening, but there is a gap between the colorimetric methods still employed in some countries and the generalised employ of MS that CE can help to solve.

Our research group developed a rapid, automated and simple method [18,19] applied to urine, using capillary electrophoresis and having a low operational cost. Moreover, it requires a very small volume of urine (50 $\mu$ l is enough) and no sample preparation. This method permits the separation, detection and even the identification in less than 15 min of a wide range of metabolic disorders related to the excretion of large amounts of organic acids in urine. It can be also applied to other body fluids, such as plasma, cerebrospinal fluid (data not shown) or amniotic liquids [20].

Although the frequency of these disorders is low, the rapid, automated, accurate analysis of organic acids, with a very small and easily transportable sample, is clinically important and very useful. The organic acids that mark these disorders are mainly detected in urine because of the rapid excretion of these water soluble acids by the kidneys. No low cost screening method that can detect a multitude of organic acidurias has yet been developed. In an in-depth study considering social and economical features, Seymour concluded that in the UK the screening of only glutaric aciduria type I which can be treated if detected just after birth, could justify the cost of screening [12]. Glutaric aciduria type I is clearly detected in the present work.

To simplify sample collection, it is necessary to have more data on the adequacy of filter paper sample collection for $\mathrm{CE}$ analysis. Therefore, we have evaluated the possibility of applying the method after specimen collection in a paper filter put on the nappy of newborns. Interferences coming from the nappy, recoveries obtained, possible correction of recoveries with creatinine and stability of the compounds have been studied. Moreover, we have optimised the media and conditions for re-extraction to give the best sensitivity and a more simple procedure.

\section{Experimental}

\subsection{CE system}

Capillary zone electrophoresis (CZE) was performed on a Beckman System 5500 (P/ACE) equipped with a UV detector set at $200 \pm 10 \mathrm{~nm}$, an automatic injector, a column cartridge pretreated with polyacrylamide (Beckman, Palo Alto, CA, USA) $37 \mathrm{~cm}$ in length, with internal diameter $50 \mu \mathrm{m}$, and the detector window was set at $30.6 \mathrm{~cm}$. All the experiments were carried out at $25^{\circ} \mathrm{C}$. Sample injections were made by pressure for $5 \mathrm{~s}$ ( 0.5 p.s.i.). Separation was developed with an applied reversed voltage $(V)$ of $10 \mathrm{kV}$.

\subsection{Chemicals and samples}

\subsubsection{Standards}

Standards, minimum 95\%, were obtained from Sigma (St. Louis, MO, USA). 


\subsubsection{Buffers}

Phosphoric acid 85\% was from Merck (Darmstadt, Germany), sodium hydroxide from Panreac (Madrid, Spain) and methanol HPLC grade from Scharlau (Barcelona, Spain).

Buffer solutions and all the dilutions were prepared with water purified by a Milli-pore Milli-QSystem (Millipore, Bedford, MA, USA). The electrophoretic buffer $\mathrm{S}, \mathrm{pH}$ 6.0, was prepared as previously described [19], that is $0.2 \mathrm{M}$ phosphoric acid adjusted to $\mathrm{pH} 6.0$ with $\mathrm{NaOH}$, and mixed with $10 \%$ $\mathrm{v} / \mathrm{v}$ of methanol.

\subsubsection{Samples}

Fresh normal urine samples from children under 8 years old were kindly provided by Virgen del Rocío Hospital (Sevilla, Spain) in compliance with the Helsinki declaration (1996). They were pooled, aliquoted and kept at $-20{ }^{\circ} \mathrm{C}$ for the optimisation study. The same hospital provided a sample from a patient with alkaptonuria, who had been previously diagnosed and it is being monitored to study the response to therapy.

A model pathological sample was prepared by spiking the normal urine with the assayed standards in the concentrations shown below.

At the end of the assay, real urine samples coming from babies under 4 months were collected by volunteer parents following the designed protocol, similar to those employed in other screening [4].

\subsubsection{Peak assignment and quantification}

Peak assignment in urine samples or paper extracts was performed by comparison of migration time with the corresponding standards and confirmed by coinjection of the standard. Quantification was accomplished by the external standard method.

\subsubsection{Creatinine}

Creatinine was measured spectrophotometrically by the Jaffé method [21].

\subsubsection{CE method}

A previous CE method [19] was optimised in order to detect a wide range of metabolic disorders related to the excretion of large amounts of organic acids in urine. Although 30 organic acids can be separated with this method, only 17 have been employed in this study, because some of them are not commercially available and some of them are very unstable. In Fig. 1, electropherogram $\mathrm{F}$ shows the separation of 17 standards of different organic acids employed in the study by $\mathrm{CE}$ using buffer $\mathrm{S}$ as electrolyte at $-10 \mathrm{kV}$ in less than $15 \mathrm{~min}$. Working concentrations are fully in the range, or sometimes under, pathological conditions considering a typical urine containing $1 \mathrm{~m} M$ in creatinine.

For the urine analysis, the samples were diluted (1:3) and centrifuged prior to the run with buffer $S$ described above. If any peak with migration time corresponding to a standard was increased, a pathology was suspected and it ought to be confirmed by co-injecting the sample (or spiking) with the suspected compound.

To facilitate the collection of urine samples before the $\mathrm{CE}$ analysis in the clinical laboratory we have also evaluated the degree of specimen impregnation employing filter paper strips located on the diaper of newborns.

\subsubsection{Optimisation of sample collection and re- extraction}

The analysed compounds are water soluble and water would be the best solvent for CZE, but it is necessary to evaluate if these acids are re-extracted in pure water from filter paper. For testing recovery, the standards $(6 \mathrm{ml})$ in concentrations around the low to medium pathological levels (Table 1) were poured onto $10 \times 6 \mathrm{~cm}$ pieces of absorbent filter paper. It was dried on a clean non-absorbent surface in room air over $24 \mathrm{~h}$. A $20-\mathrm{cm}^{2}$ section was cut from the paper, fan folded, and placed in a disposable 12-ml polyethylene tube. The soluble components were eluted with $3 \mathrm{ml}$ of pure water; after addition of water, the tube was mechanically shaken for $5 \mathrm{~min}$, allowed to stand for $15 \mathrm{~min}$ and then the tube was centrifuged for $15 \mathrm{~min}$ at $2000 \mathrm{~g}$ and processed as described below.

A $500-\mu 1$ aliquot was removed for creatinine determination.

Two different brands of filter paper were tested to evaluate differences in sample retention or possible interferences in the electropherograms.

In the same way, three different brands of diapers were tested: one of them sold in pharmacy shops (mainly consisting of cellulose) and the other two 
were known mass market brands (with a more complex composition), all of them for babies under 6 months.

For the optimisation of some preliminary parameters: solvent extraction volume, paper type and diaper, etc., some representative acids of the group were chosen. The assay was performed with ketoisovaleric and orotic acids, representatives of ketoacids and aromatic acids, and lactic as an aliphatic acid. Creatinine was also included.

For the optimization of the eluant volume, nine strips of filter paper $2 \times 10 \mathrm{~cm}$ were impregnated with $2 \mathrm{ml}$ of standard solution containing ketoglutaric acid, lactic acid, ketoisovaleric acid, pyruvic acid, orotic acid and creatinine.

The extraction of the acids was carried out with 2 , 3 and $4 \mathrm{ml}$ of Milli-Q water, in triplicate. The extraction process was carried out as previously described.

The stability of these acids on dried filter paper stored at room temperature was examined by spiking filter papers $(6 \times 10 \mathrm{~cm})$ with $6 \mathrm{ml}$ of urine with all the standards added and assaying them after different storage times throughout 1 month, as compared with the spiked urine measured directly.

Simulation of the absorption from a diaper in the whole process involved application of the urine spiked with all the acids to a filter paper put on a diaper. It was compared with the same sample poured on a filter paper over a non-absorbent surface.

Optimised conditions for sample collection and processing were: absorbent filter paper $10 \times 6 \mathrm{~cm}$ was put on the baby's diaper and closed. After wetting, it was allowed to dry on a clean, flat, non-absorbent surface in room air and after $24 \mathrm{~h}$ it was sent to the laboratory. There, strips of $2 \times 10 \mathrm{~cm}$ were cut, one of them was fan folded and placed in a disposable 12-ml polyethylene tube. The soluble components were eluted with $3 \mathrm{ml}$ of pure water by mechanical shaking for $5 \mathrm{~min}$. Then, the tube was centrifuged for $15 \mathrm{~min}$ and processed as previously described. Analysis ought to be performed within 2 weeks and creatinine measured to find out urine concentration and impregnation degree.

\subsubsection{Quantification}

The peak areas of the acids were compared with those obtained from identical concentrations of acids without prior extraction. When samples of urine were spiked with standard acids the samples were previously quantified to determine the natural existing acids.

The whole method was applied to normal urine samples by giving the papers and adequate instructions to the parents of the children.

A urine sample of a patient with alkaptonuria was directly measured and then it was impregnated following the procedure previously described. Samples were re-extracted and measured after 1,7 and 14 days.

\section{Results and discussion}

Recoveries of the acids and creatinine with MilliQ water from filter paper are shown in Table 1 for 12 different strips. Most of the acids have recoveries near $100 \%$, considering the limits of confidence. Acetoacetic and propionic acids have lower recoveries because they are highly volatile, while homogentisic acid is easily oxidizable. Although extraction of creatinine is not optimal, water can be

Table 1

Organic acids and creatinine recovery from filter paper with water

\begin{tabular}{llll}
\hline Acid & $\begin{array}{l}\text { Concentration } \\
(\mathrm{m} M)\end{array}$ & $\begin{array}{l}\text { \% Organic acid } \\
\text { recovery } \pm \text { C.L. }\end{array}$ & $n$ \\
\hline Oxalic & 0.24 & $75 \pm 12$ \\
Fumaric & 0.24 & $93 \pm 16$ \\
Ketoglutaric & 0.36 & $80 \pm 13$ & \\
Malic & 1.50 & $89 \pm 15$ & \\
Methylmalonic & 0.36 & $87 \pm 13$ & \\
Glutaric & 0.36 & $87 \pm 15$ & \\
Pyruvic & 1.20 & $91 \pm 17$ & \\
$N$-Acetyl-L-aspartic & 2.40 & $87 \pm 15$ & \\
Glycolic & 0.72 & $86 \pm 16$ & \\
Acetoacetic & 0.48 & $68 \pm 12$ & \\
Propionic & 4.00 & $9 \pm 2$ & \\
Lactic & 2.40 & $94 \pm 17$ & \\
Ketoisovaleric & 0.72 & $86 \pm 15$ & \\
3-Hydroxybutyric & 1.44 & $87 \pm 14$ & \\
Pyroglutamic & 0.64 & $94 \pm 20$ & \\
DL-Hydroxyisovaleric & 2.04 & $81 \pm 14$ & \\
Orotic & 0.12 & $96 \pm 17$ & \\
Phenyllactic & 0.16 & $89 \pm 19$ & \\
Homogentisic & 0.16 & $69 \pm 15$ & \\
Hippuric & 0.05 & $91 \pm 18$ & \\
& & $76 \pm 14$ & \\
Creatinine & 0.80 & & \\
\hline & & & \\
\hline
\end{tabular}


considered a good solvent for extraction and it is the best for the analytical technique.

The only notable peak observed on blank runs, where de-ionized water had been directly applied to both types of filter paper, was an unknown whose migration time did not interfere with the analytes (Fig. 1). No difference was found in the absorbent power either. The rest of the study was performed with Whatman paper.

The same was observed with the three brands of diapers, whose blanks are also shown in Fig. 1. Moreover, there is no statistical difference in the recoveries obtained from the two filter papers and the three diapers in the assayed acids (Student $t$-test, $P>95 \%)$. Therefore, one of them was randomly selected for the rest of the assay: Whatman filter paper and a well-known mass market diaper brand.

For the comparison of the recoveries of each acid with the three different water volumes $(2,3$ and 4 $\mathrm{ml})$, one-way ANOVA was applied $(P>95 \%)$ and no statistical differences appeared.

Three $\mathrm{ml}$ was considered the most appropriate volume because it is easy to manipulate and in such conditions the final dilution of the acids would be very similar to that employed in the proposed $\mathrm{CE}$ method for urine samples [19].

Table 2 shows the recoveries of all the standards and creatinine when the filter paper was put on a diaper. Obviously recoveries decreased, as part of the sample goes into the diaper. Recoveries of the acids were near $45 \%$, except the highly volatile (propionic and acetoacetic) or oxidizable (homogentisic) ones. Creatinine recovery is always lower and therefore recoveries may be over $100 \%$ when expressed by creatinine.

Table 3 shows the values obtained for the quantifi-

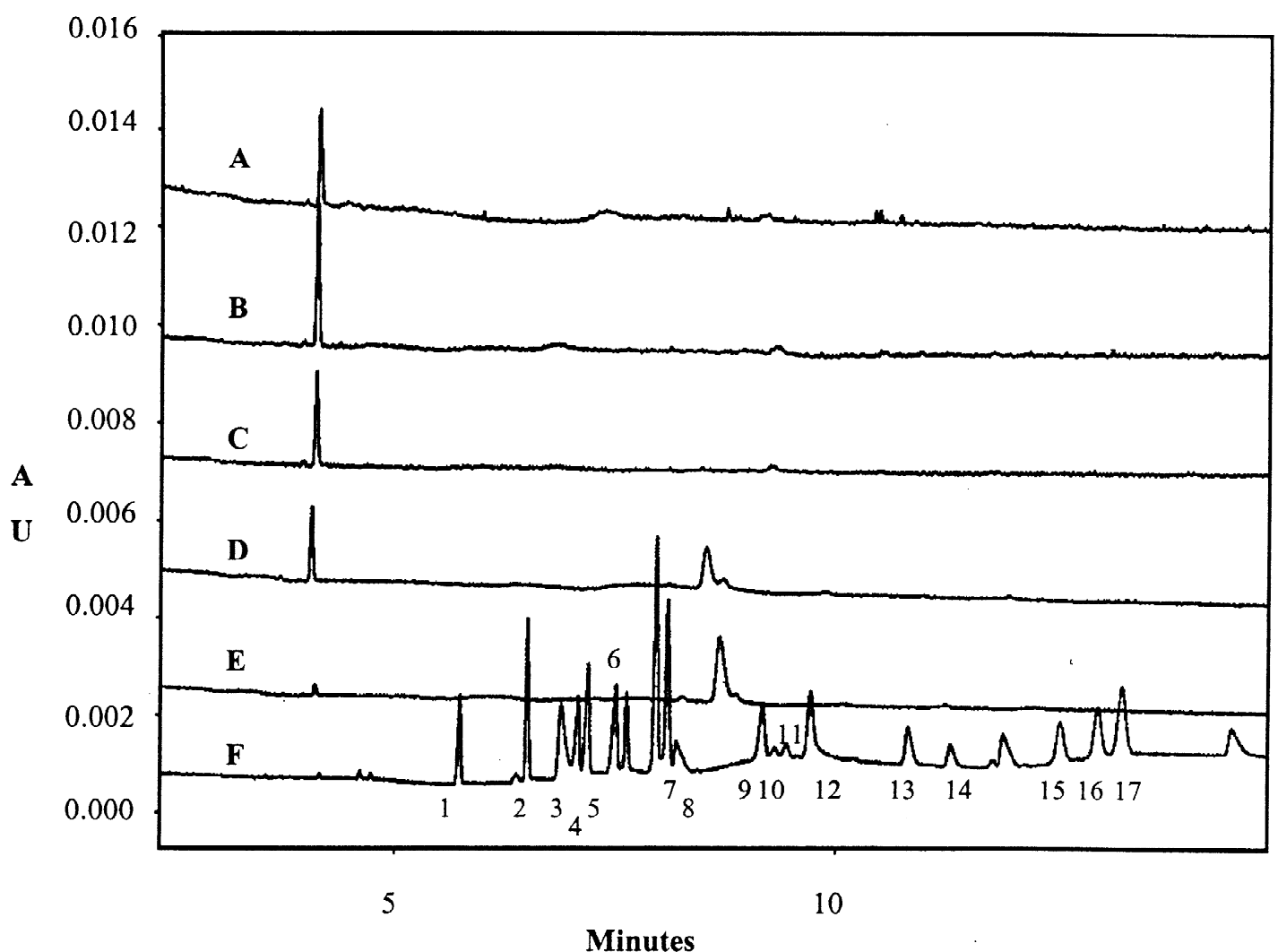

Fig. 1. Electropherograms from: (A) diaper type A; (B) diaper type B; (C) diaper type C; (D) Whatman filter paper; (E) Schleicher \& Schuell; (F) standards of organic acids: 1, oxalic; 2, fumaric; 3, ketoglutaric; 4, malic; 5, methylmalonic; 6, glutaric; 7, $N$-acetyl-L-aspartic; 8, glycolic; 9, acetoacetic; 10, propionic; 11, lactic; 12, ketoisovaleric; 13, 3-hydroxybutyric; 14, DL-hydroxyisovaleric; 15, phenyllactic; 16, homogentisic; 17, hippuric. 
Table 2

Organic acid standards and creatinine recovery from filter paper put on a diaper

\begin{tabular}{|c|c|c|}
\hline Acids & $\begin{array}{l}\% \text { Organic acid } \\
\text { recovery } \pm \text { C.L. }\end{array}$ & $\begin{array}{l}\% \text { Organic acid recovery } \\
\text { with creatinine } \pm \text { C.L. }\end{array}$ \\
\hline Oxalic & $37 \pm 5$ & $124 \pm 11$ \\
\hline Fumaric & $48 \pm 6$ & $164 \pm 10$ \\
\hline Ketoglutaric & $42 \pm 5$ & $141 \pm 8$ \\
\hline Malic & $45 \pm 5$ & $152 \pm 8$ \\
\hline Methylmalonic & $46 \pm 5$ & $154 \pm 6$ \\
\hline Glutaric & $45 \pm 5$ & $152 \pm 10$ \\
\hline Pyruvic & $43 \pm 6$ & $111 \pm 12$ \\
\hline$N$-Acetyl-L-aspartic & $45 \pm 6$ & $153 \pm 11$ \\
\hline Glycolic & $50 \pm 6$ & $169 \pm 16$ \\
\hline Acetoacetic & $27 \pm 4$ & $91 \pm 8$ \\
\hline Propionic & $4.7 \pm 0.5$ & $16 \pm 2$ \\
\hline Lactic & $45 \pm 6$ & $150 \pm 15$ \\
\hline Ketoisovaleric & $45 \pm 6$ & $153 \pm 15$ \\
\hline 3-Hydroxybutyric & $44 \pm 6$ & $151 \pm 24$ \\
\hline Pyroglutamic & $42 \pm 4$ & $90 \pm 11$ \\
\hline DL-Hydroxyisovaleric & $45 \pm 6$ & $151 \pm 18$ \\
\hline Orotic & $45 \pm 5$ & $118 \pm 12$ \\
\hline Phenyllactic & $42 \pm 5$ & $96 \pm 11$ \\
\hline Homogentisic & $28 \pm 6$ & $72 \pm 11$ \\
\hline Hippuric & $42 \pm 7$ & $107 \pm 11$ \\
\hline$\%$ Creatinine recovery \pm L.C. & $34 \pm 5$ & \\
\hline
\end{tabular}

Table 3

Concentrations of organic acids added to a urine sample in filter paper and measured on different days

\begin{tabular}{|c|c|c|c|c|c|}
\hline & \multirow{2}{*}{$\begin{array}{l}\text { Concentration } \\
\mathrm{S}+\mathrm{U}\end{array}$} & \multicolumn{4}{|l|}{$(\mathrm{m} M) \pm \mathrm{C} . \mathrm{L}$} \\
\hline & & $24 \mathrm{~h}$ & 7 days & 15 days & 30 days \\
\hline Oxalic & $0.08 \pm 0.02$ & $0.1 \pm 0.5$ & $0.17 \pm 0.06$ & $0.21 \pm 0.07$ & $0.2 \pm 0.1$ \\
\hline Fumaric & $0.23 \pm 0.01$ & $0.33 \pm 0.07$ & $0.30 \pm 0.08$ & $0.28 \pm 0.03$ & - \\
\hline Ketoglutaric & $1.24 \pm 0.04$ & $1.5 \pm 0.3$ & $1.0 \pm 0.3$ & $0.9 \pm 0.1$ & $0.2 \pm 0.1$ \\
\hline Malic & $1.4 \pm 0.1$ & $1.7 \pm 0.4$ & $1.9 \pm 0.5$ & $2.2 \pm 0.2$ & $0.18 \pm 0.06$ \\
\hline Methylmalonic & $0.68 \pm 0.09$ & $0.9 \pm 0.3$ & $0.9 \pm 0.3$ & $1.1 \pm 0.1$ & $0.5 \pm 0.2$ \\
\hline Glutaric & $1.21 \pm 0.04$ & $1.7 \pm 0.3$ & $1.6 \pm 0.4$ & $1.8 \pm 0.2$ & $1.5 \pm 0.9$ \\
\hline$N$-Acetyl-L-aspartic & $3.67 \pm 0.02$ & $5 \pm 1$ & $4 \pm 1$ & $4.9 \pm 0.5$ & $7 \pm 2$ \\
\hline Lactic & $4.1 \pm 0.8$ & $3.1 \pm 0.8$ & $2.9 \pm 0.6$ & $2.6 \pm 0.3$ & - \\
\hline Ketoisovaleric & $0.48 \pm 0.05$ & $0.8 \pm 0.2$ & $0.50 \pm 0.08$ & - & - \\
\hline 3-Hydroxybutyric & $4.8 \pm 0.4$ & $6 \pm 1$ & $6 \pm 2$ & $7 \pm 1$ & $2.9 \pm 0.8$ \\
\hline Pyroglutamic & $1.16 \pm 0.06$ & $1.0 \pm 0.3$ & $1.0 \pm 0.3$ & $1.2 \pm 0.4$ & $1.1 \pm 0.2$ \\
\hline Hydroxyisovaleric & $2.7 \pm 0.2$ & $3.9 \pm 0.8$ & $3 \pm 1$ & $3.2 \pm 0.3$ & $2.6 \pm 0.5$ \\
\hline Orotic & $0.2 \pm 0.01$ & $0.19 \pm 0.05$ & $0.13 \pm 0.03$ & $0.18 \pm 0.05$ & $0.17 \pm 0.03$ \\
\hline Phenyllactic & $0.57 \pm 0.04$ & $0.5 \pm 0.1$ & $0.5 \pm 0.1$ & $0.5 \pm 0.2$ & $0.6 \pm 0.1$ \\
\hline Homogentisic & $0.1 \pm 0.03$ & $0.07 \pm 0.02$ & $0.05 \pm 0.02$ & $0.13 \pm 0.04$ & $0.04 \pm 0.01$ \\
\hline Hippuric & $8.8 \pm 0.5$ & $8 \pm 2$ & $8 \pm 2$ & $9 \pm 2$ & $8 \pm 1$ \\
\hline
\end{tabular}

$\mathrm{S}+\mathrm{U}$, standards + urine.

cation of the urine sample spiked with all the assayed acids measured directly and after being impregnated in filter paper and maintained at room temperature for $1,8,14$ and 28 days. $p$-Hydroxyphenylpyruvic and phenylpyruvic acids were not included because their instability is well-known and the degradation 
Table 4

Organic acids found as naturally occurring in a urine pool from healthy children

\begin{tabular}{lc}
\hline Organic acid & $\mathrm{mmol} / \mathrm{mol} \mathrm{Cr} \pm$ C.L. $(n=8)$ \\
\hline Oxalic & $24 \pm 10$ \\
Ketoglutaric & $326 \pm 13$ \\
$N$-Acetyl-L-aspartic & $9 \pm 1$ \\
Pyroglutamic & $65 \pm 5$ \\
Phenyllactic & $64 \pm 5$ \\
Hippuric & $518 \pm 41$ \\
Creatinine & $5 \pm 2$ \\
\hline
\end{tabular}

products can interfere with the analysis. If, in some case, one of these analytes, $p$-hydroxyphenylpyruvic or phenylpyruvic acid, were present in a pathological sample, it would be detected, due to their high UV absorption, in spite of being partially degraded. High values for some of the standard deviations in this table and some specific increases of concentration values were not due to the analytical method, but to the collection procedure. When wet paper is not left completely flat to let dry, acids migrate irregularly and these differences appear. That is one of the arguments for measuring systematically creatinine in the strips.

In order to evaluate the stability, concentrations of each acid were represented versus time. The acids whose slopes with the confidence limits $(P>95 \%)$ include the zero value were considered stable. Glutaric, pyroglutamic, orotic, phenyllactic, and hippuric and orotic acids are stable in these conditions over 30 days. Methylmalonic, $N$-acetylaspartic, lactic, 3-hydroxybutyric, hydroxyisovaleric and fumaric acids are stable for 15 days. Ketoglutaric, fumaric, malic and ketoisovaleric are stable for 7 days.

Homogentisic acid concentration, easily oxidizable, decreases quickly, but it is usually high in pathological samples and therefore easily detectable in spite of the decrease. Glycolic, acetoacetic and propionic acids, highly volatile, are not even detected

Table 5

Recovery of organic acids added to urine from filter paper without and with diaper and expressed or not by creatinine

\begin{tabular}{|c|c|c|c|c|}
\hline \multirow[t]{2}{*}{ Acids } & \multicolumn{2}{|l|}{ Without diaper } & \multicolumn{2}{|l|}{ With diaper } \\
\hline & $\begin{array}{l}\% \text { Organic acid } \\
\text { recovery } \pm \text { C.L. }\end{array}$ & $\begin{array}{l}\text { \% Organic acid recovery } \\
\text { with } \mathrm{Cr} \pm \text { C.L. }\end{array}$ & $\begin{array}{l}\% \text { Organic acid } \\
\text { recovery } \pm \text { C.L. }\end{array}$ & $\begin{array}{l}\% \text { Organic acid recovery } \\
\text { with } \mathrm{Cr} \pm \text { C.L. }\end{array}$ \\
\hline Oxalic & $41 \pm 9$ & $39 \pm 4$ & $69 \pm 5$ & $151 \pm 17$ \\
\hline Fumaric & $113 \pm 18$ & $112 \pm 13$ & $59 \pm 5$ & $127 \pm 7$ \\
\hline Ketoglutaric & $111 \pm 17$ & $109 \pm 7$ & $46 \pm 4$ & $101 \pm 5$ \\
\hline Malic & $117 \pm 20$ & $114 \pm 9$ & $49 \pm 6$ & $106 \pm 8$ \\
\hline Methylmalonic & $109 \pm 21$ & $106 \pm 14$ & $41 \pm 6$ & $88 \pm 8$ \\
\hline Glutaric & $114 \pm 17$ & $112 \pm 5$ & $46 \pm 3$ & $99 \pm 6$ \\
\hline Pyruvic & - & - & - & - \\
\hline$N$-Acetyl-L-aspartic & $124 \pm 20$ & $121 \pm 8$ & $50 \pm 4$ & $109 \pm 7$ \\
\hline Glycolic & - & - & - & - \\
\hline Acetoacetic & - & - & - & - \\
\hline Propionic & $21 \pm 4$ & $23 \pm 6$ & - & - \\
\hline Lactic & $121 \pm 19$ & $118 \pm 5$ & $77 \pm 12$ & $167 \pm 19$ \\
\hline Ketoisovaleric & $130 \pm 18$ & $128 \pm 7$ & $60 \pm 4$ & $132 \pm 12$ \\
\hline 3-Hydroxybutyric & $145 \pm 24$ & $147 \pm 11$ & $56 \pm 4$ & $122 \pm 10$ \\
\hline Pyroglutamic & $88 \pm 11$ & $72 \pm 8$ & $40 \pm 4$ & $90 \pm 11$ \\
\hline DL-Hydroxyisovaleric & $124 \pm 19$ & $121 \pm 6$ & $47 \pm 5$ & $103 \pm 10$ \\
\hline Orotic & $86 \pm 9$ & $70 \pm 6$ & $40 \pm 3$ & $89 \pm 9$ \\
\hline Phenyllactic & $85 \pm 10$ & $69 \pm 7$ & $42 \pm 4$ & $96 \pm 11$ \\
\hline Homogentisic & $52 \pm 6$ & $42 \pm 4$ & $39 \pm 3$ & $120 \pm 13$ \\
\hline Hippuric & $87 \pm 9$ & $71 \pm 7$ & $37 \pm 6$ & $87 \pm 17$ \\
\hline \multicolumn{2}{|c|}{$\%$ Creatinine recovery \pm C.L. } & $110 \pm 13$ & & $45 \pm 4$ \\
\hline
\end{tabular}

Cr., creatinine. 
at 8 days. Lactic and fumaric acid signals completely disappeared at 28 days.

Therefore, measurement is recommended within 2 weeks, as most of the assayed organic acids are stable at 14 days.

Finally a urine pool was quantified. The acids shown in Table 4 were found as naturally occurring. This urine was spiked with all the assayed acids and recoveries from filter paper put on a nonabsorbent surface or on a diaper were calculated. Data are collected in Table 5. Results and therefore conclusions are quite similar to those obtained during optimization for standards.

Once conditions were optimized, real urine samples from healthy babies $(n=6)$ collected by their parents were studied. Fig. 2 shows one of these urine extracts. The peaks identified corresponded to oxalic, ketoglutaric, $\mathrm{N}$-acetylaspartic, pyroglutamic, orotic, phenyllactic and hippuric acids. Peaks were identified by migration times as compared with standards and by co-injection with the suspected compound. The standards were divided in two groups to make the identification easier. The organic acids found in these urine samples were under pathological levels. Samples contaminated with feces or creams did not interfere with the analysis.

The alkaptonuria urine sample gave a concentration of $1030 \mathrm{~m} M$ homogentisic acid $/ \mathrm{mol}$ creatinine directly measured. After being impregnated in filter paper for $24 \mathrm{~h}$ and re-extracted, the value was $846 \mathrm{mM} / \mathrm{mol}$ creatinine $(82 \%$ of the initial value), after 7 days it was $741 \mathrm{~m} M / \mathrm{mol}$ creatinine (72\%) and after 14 days it was $774 \mathrm{mM} /$ mol creatinine $(75 \%)$. Fig. 3 shows the electropherograms corresponding to homogentisic acid standard $50 \mu M$ and the different paper extracts at 24

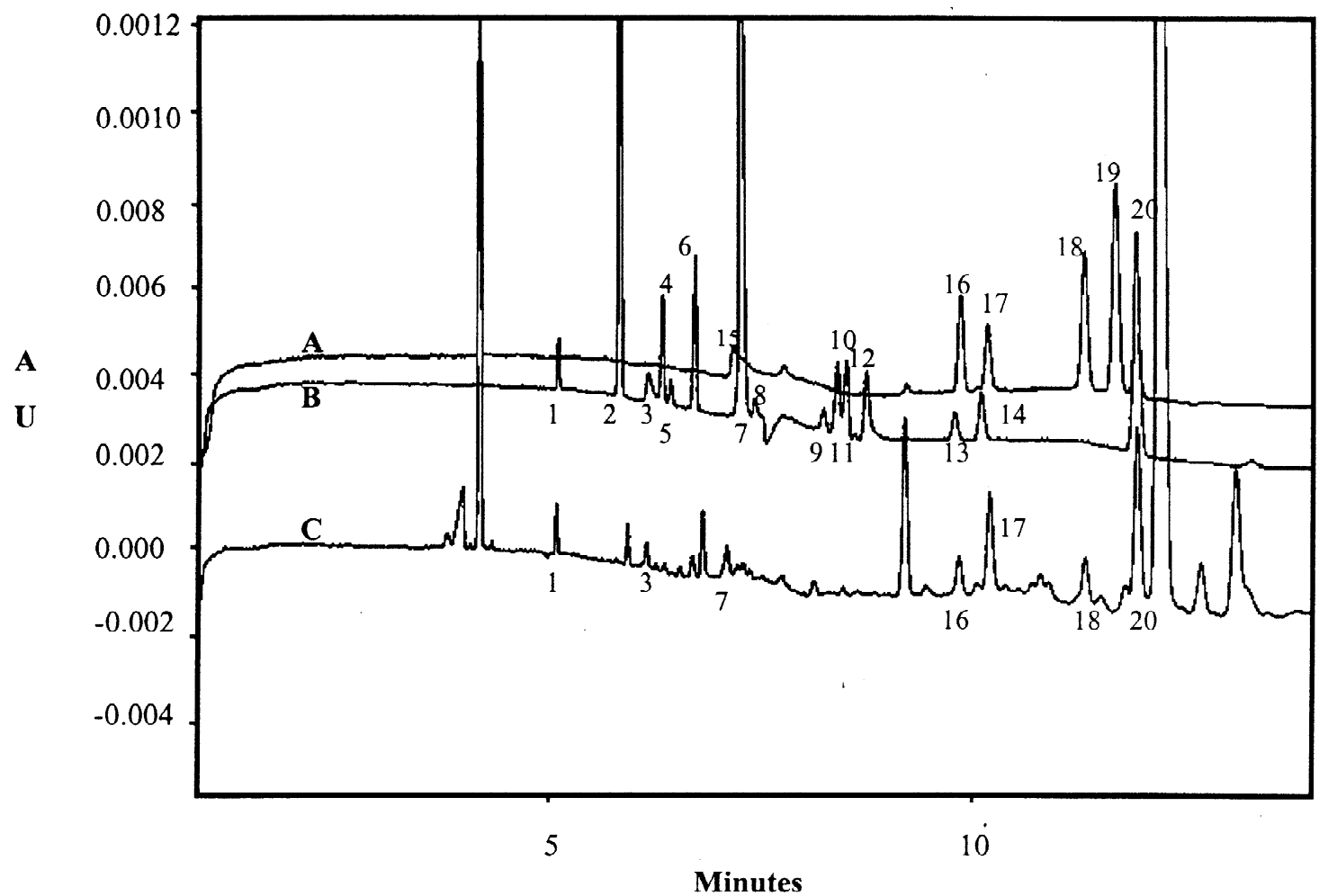

Fig. 2. Electropherogram from: (A) standard mixture I; (B) standard mixture II; (C) urine extract. Peak identification: 1, oxalic; 2, fumaric; 3, ketoglutaric; 4, malic; 5, methylmalonic; 6, glutaric; 7, $N$-acetyl-L-aspartic; 8, glycolic; 9, acetoacetic; 10, propionic; 11, lactic; 12, ketoisovaleric; 13, 3-hydroxybutyric; 14, DL-hydroxyisovaleric; 15, pyruvic; 16, pyroglutamic; 17, orotic; 18, phenyllactic; 19, homogentisic; 20, hippuric. 


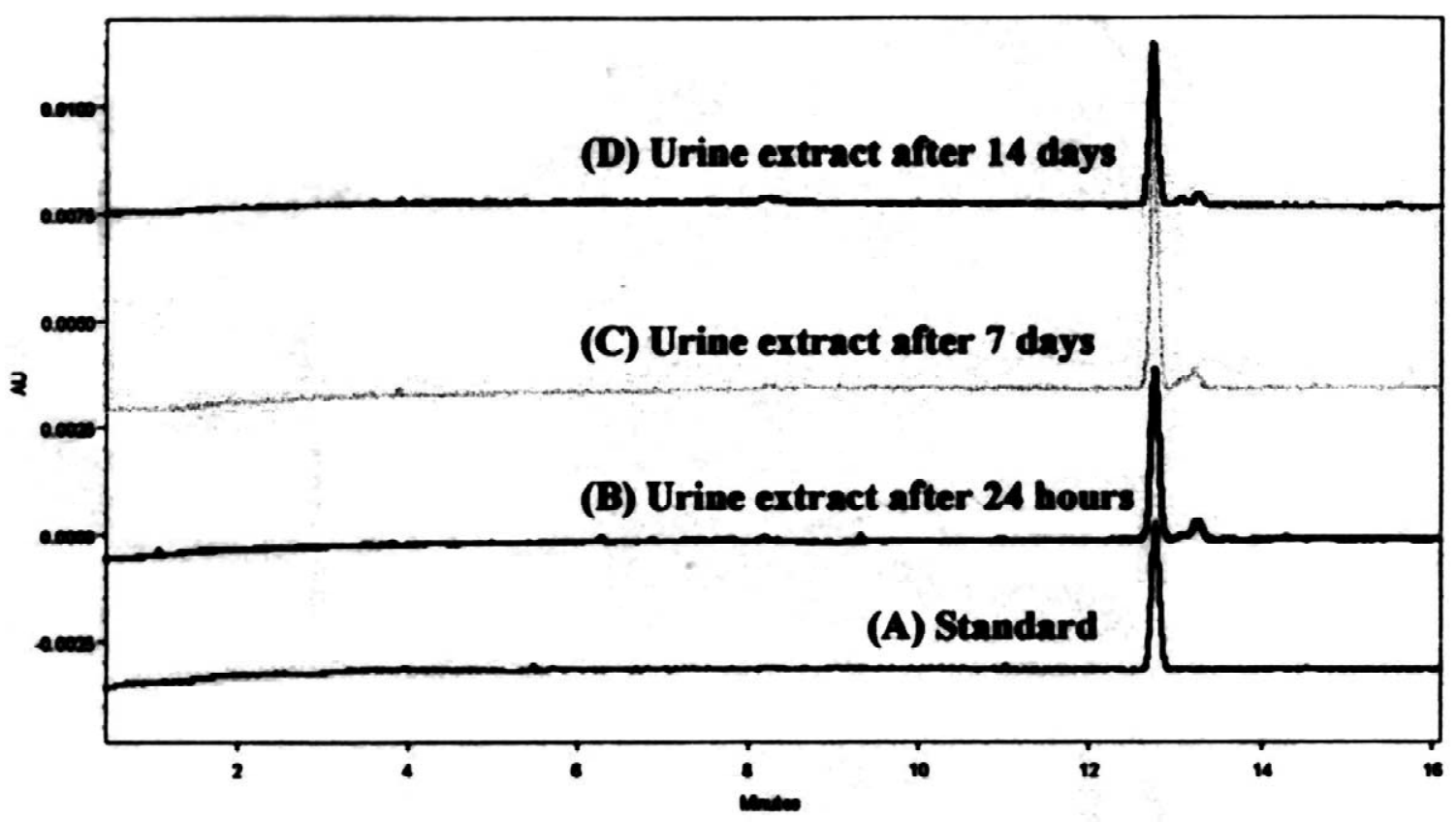

Fig. 3. Electropherogram from: (A) homogentisic acid standard $50 \mu M$; (B) alkaptonuria urine sample extracted after $24 \mathrm{~h}$ in filter paper; (C) the same urine after 7 days in filter paper; (D) after 14 days.

h, 7 days and 14 days. Even considering oxidation and all the experimental errors associated to the extraction process, identification is unquestionable and quantification is very good.

\section{Conclusion}

The method we report has the advantages of easy sample collection, easy shipping or delivery, and rapid analysis. It could be an important point for families living far away from hospitals or in countries where deliveries are mainly at home. Moreover, this method of collection and analysis can be used for those acids, such as glutaric, for which screening is considered especially advisable [12].

$\mathrm{CE}$ analysis could achieve diagnostically accurate results of many metabolic disorders after elution of urine from the filter paper.

The real incidence of organic acidurias detectable by this screening method is unknown. The infrastructure for statewide urine collection from babies could be the same as for mass blood screening for phenylketonuria, but with different resources for analysis. Finally, another strip of the same filter paper is suitable for further analysis, when an abnormal acid profile is detected, and a deeper knowledge or identification of analytes is needed by GC-MS or other techniques.

\section{Acknowledgements}

The authors thank Dr. Gayoso for his encouragement in the execution of the present work and C.A.M. project no. 08.6/0001.1/99 and San PabloCEU University project no. 3/99 for financial support.

\section{References}

[1] Y. Kinoshita, S. Yamada, K. Haraguchi, T. Takayanagi, Y. Mori, T. Takahashi, E. Haruki, Clin. Chem. 34 (1998) 2228.

[2] R. Kerbl, C.E. Urban, R. Ladenstein, I.M. Ambros, E. Spuller, I. Mutz, G. Amann, H. Kovar, H. Gadner, P.F. Ambros, Med. Pediatr. Oncol. 29 (1997) 1.

[3] J.L. Ater, K.L. Gardner, L.E. Foxhall, B.L. Therrell Jr., W.A. Bleyer, Cancer 82 (1998) 1593. 
[4] M. Tuchman, B. Lemieux, C. Auray-Blais, L.L. Robison, R. Giguere, M.T. McCann, W.G. Woods, Pediatrics 86 (1990) 765.

[5] J.A. Seviour, A.C. McGill, A.W. Craft, L. Parker, S. Bell, M. Cole, J. Smith, E. Hawkins, J. Brown, A.D. Gordon, Am. J. Pediatr. Hematol./Oncol. 14 (1992) 332.

[6] J. Hanai, T. Kawai, Y. Sato, N. Takasugi, M. Nishi, T. Takeda, Clin. Chem. 33 (1987) 2043.

[7] M.T. McCann, M.M. Thompson, I.C. Gueron, B. Lemieux, R. Giguère, M. Tuchman, Clin. Chem. 42 (1996) 910.

[8] B. Lemieux, C. Auray-Blais, R. Giguère, D. Shapcot, C.R. Scriver, J. Inherit. Metab. Dis. 11 (1998) 45.

[9] M.T. McCann, M.M. Thompson, I.C. Gueron, M. Tuchman, Clin. Chem. 41 (1995) 739.

[10] M. Tuchman, M.T. McCann, P.E. Johnson, B. Lemieux, Pediatr. Res. 30 (1991) 315.

[11] B.A. Chamberlin, C.C. Sweeley, Clin. Chem. 33-34 (1987) 572.
[12] C.A. Seymour, M.J. Thomason, R.A. Chalmers, G.M. Addison, M.D. Bain, F. Cockburn, P. Littlejohns, J. Lord, A.H. Wilcox, in: Health Technology Assessment Programme, Vol. 1, 1997, p. 1.

[13] H.L. Levy, Clin. Chem. 44 (1998) 2401.

[14] R. Lehmann, W. Voelter, H.M. Liebich, J. Chromatogr. B 697 (1997).

[15] K. Kitagishi, H. Shintani, J. Chromatogr. B 717 (1998) 327.

[16] T. Adam, D. Friedecký, L.D. Fairbanks, J. Sevcík, P. Barták, Clin. Chem. 45 (1999) 2086.

[17] C. Blessum, J.O. Jeppsson, F. Aguzzi, H. Bernon, J. Bienvenu, Ann. Biol. Clin. 57 (1999) 643.

[18] C. Barbas, N. Adeva, R. Aguilar, M. Rosillo, T. Rubio, M. Castro, Clin. Chem. 44 (1998) 1340.

[19] A. García, C. Barbas, R. Aguilar, M. Castro, Clin. Chem. 44 (1998) 1905

[20] L. Saavedra, C. Barbas, J. Chromatogr. B 766 (2002) 235.

[21] M. Jaffé, Z. Physiol. Chem. 10 (1886) 391. 